\title{
SMOOTH NONTRIVIAL 4-DIMENSIONAL $s$-COBORDISMS
}

\author{
SYLVAIN E. CAPPELL AND JULIUS L. SHANESON
}

\begin{abstract}
This announcement exhibits smooth 4-dimensional manifold triads $\left(W ; M_{0}, M_{1}\right)$ which are s-cobordisms, i.e. the inclusions $M_{i} \subseteq W, i=0,1$, are simple homotopy equivalences, but are not diffeomorphic or even homeomorphic to a product $M_{i} \times[0,1]$.
\end{abstract}

The Barden-Mazur-Stallings $s$-cobordism theorem constitutes one of the foundational stones of modern topology. It asserts, in the smooth, piecewiselinear, or topological categories, that if $W$ is a manifold of dimension at least six, with boundary components $M_{i}, i=0,1$, whose inclusions into $W$ are simple homotopy equivalences, then $W$ is necessarily a product (see $[\mathbf{K}, \mathbf{H}$, RS, KS]). For simply connected smooth manifolds of dimension at least six, this result had already been proven by Smale as the " $h$-cobordism theorem" [Sm], with the generalized Poincaré conjecture in higher dimensions as a corollary. The $s$-cobordism statement holds in dimensions one and two, and is equivalent to the Poincaré conjecture in dimension three. Freedman [F1, F2] proved the five-dimensional result for topological manifolds with fundamental group of polynomial growth (e.g. finite or polycyclic). Donaldson's extraordinary results imply the failure of the five-dimensional result in the smooth (or piecewise linear) category even for simply connected manifolds; by [F1] the resulting $h$-cobordisms will still be topological products. Using Freedman's results, the present authors produced some nontrivial orientable four-dimensional topological $s$-cobordisms [CS1, CS2]. (See [MS] for a nonorientable and definitely nonsmoothable example.) These topological constructions have been further studied and extended by Kwasik and Schultz [KwS].

We will now use a different construction to produce some nontrivial smooth $s$-cobordisms. Neither the construction nor the proof rely on any of the results cited above. Let $M$ be a quaternionic space-form; i.e.

$$
M=M_{r}=S^{3} / Q_{r},
$$

$Q_{r}$ the quaternionic group of order $2^{r+2}$. Then it is well known that the orientable manifold $M$ has a one-sided Heegaard splitting

$$
M=N(K) \cup H,
$$

where $N(K)$ is the total space of an interval bundle over the Klein bottle $K$ and $H$ is a solid torus. Let $E_{0}$ be a closed tubular neighborhood of

Received by the editors January 19, 1987.

1980 Mathematics Subject Classification (1985 Revision). Primary 57R80.

Both authors partially supported by NSF grants.

(C) 1987 American Mathematical Society $0273-0979 / 87 \$ 1.00+\$ .25$ per page 
$K=K \times\{0\}$ in $M \times(-1,1)$. Then $E_{0}$ is a linear $D^{2}$-bundle over $K$ with boundary the double of $N(K)$. Let

$$
X=M \times[-1,1]-\operatorname{Int} E_{0} .
$$

The smooth $s$-cobordisms will be of the form

$$
W=W_{r}=X \cup_{\partial E_{0}} E
$$

where $E$ will be a locally trivial smooth fiber-bundle over $K$ with fiber $T_{0}^{2}=$ $S^{1} \times S^{1}-$ Int $D^{2}$, with $\partial E=\partial E_{0}$.

In fact, view $S^{1} \subset \mathbf{C}$ and define $\psi_{i}, i=1,2$, by

$$
\psi_{1}(x, y)=(y, y x) \text { and } \psi_{2}(x, y)=\left(y^{-1}, y^{-1} x^{-1}\right) .
$$

Note that $\psi_{1}^{2}=\psi_{2}^{2}$. The Klein bottle $K$ is the union of two Möbius bands, and it follows that there is a canonical $T^{2}$-bundle $E_{1}$ over $K$ whose restrictions to the cores of the Möbius bands have monodromies $\psi_{1}$ and $\psi_{2}$ respectively. Since $\psi_{i}(1,1)=(1,1)$, this bundle has a cross-section, and there is a canonical way to identify a tubular neighborhood of its image with $E_{0}$. We then take $E=E_{1}-$ Int $E_{0}$ in the above definition of $W$. Clearly, $W$ is an orientable smooth 4-manifold with two copies of $M=S^{3} / Q_{r}$ as boundary.

THEOREM. 1. The smooth four-manifold $W$ is an s-cobordism of $M$ to itself.

2. $W$ is not diffeomorphic or even homeomorphic to a product $M \times[-1,1]$.

It can also be shown that $W$ is not homeomorphic to any of the topological $s$-cobordisms of [CS2], and the smoothability of any of them remains open.

The proof of 1 uses Van Kampen's theorem and other well-known arguments in homotopy and simple homotopy theory. However, note that the restriction of a suitable diffeomorphism of $T^{2}$ isotopic to $\psi_{i}$ represents a square-root of the monodromy of the figure-eight knot.

We indicate the proof of 2 for the case $r=1$, the quaternion group of order eight. Let $P$ be obtained from $W$ by identifying $M \times\{-1\}$ with $M \times\{1\}$. Then we explicitly construct a framed 5 -manifold $U$ with the following properties:

1. $\partial U=P$.

2. There is a retraction $r: U \rightarrow M$ inducing isomorphisms on fundamental groups and homology with $\mathbf{Z}_{2}$ coefficients.

3. If $U_{4}$ and $U_{8}$ are the 4 -fold and 8-fold covers of $U$, respectively, then $\left|H_{2}\left(U_{8}\right)\right|\left|H_{2}\left(U_{4}\right)\right|^{-1} \equiv \pm 7(\bmod 16)$.

By contrast, we show that were $W$ a product and $U$ as above satisfying 1 and 2 , the quotient (of odd integers) in 3 would necessarily be congruent to $\pm 1(\bmod 16)$. Because of the possible choices for $P$ and $r$, the proof is somewhat involved. It uses the fact, due independently to J. H. Rubinstein $[\mathbf{R}]$ and the present authors, that a diffeomorphism or homeomorphism of $M$ homotopic to the identity will necessarily be isotopic to it. In the course of the proof, the remaining ambiguity of $[\mathbf{K w S}]$ concerning the classification of topological $s$-cobordisms of $M$ to itself is resolved, and a remark in [CS2] is corrected. 
It would be interesting to know if the universal covering space of $W$ is diffeomorphic to $S^{3} \times[0,1]$. This is similar to the situation for the exotic $\mathbf{R P}^{4}$ of [CS3], whose covering space is also potentially exotic [AK]. It is also of interest to observe that for the case $r=1, W$ can be embedded as a codimension zero submanifold of a smooth homotopy 4-sphere.

\section{REFERENCES}

[AK] S. Akbulut and R. C. Kirby, A potential smooth counterexample in dimension four to the Poincaré conjecture, the Schoenfliess conjecture, and the Andrews-Curtis conjecture, Topology 24 (1985), 375-390.

[CS1] S. E. Cappell and J. L. Shaneson, A counterexample to the oozing problem for closed manifolds, Lecture Notes in Math., vol. 763, Springer-Verlag, New York, 1979, pp. 627-634.

[CS2] _ On 4-dimensional s-cobordisms, J. Differential Geom. 22 (1985), 97-115.

[CS3] _ Some new 4-manifolds, Ann. of Math. (2) 104 (1976), 61-72.

[D] S. Donaldson, On Dolgachev's surfaces (to appear).

[F1] M. Freedman, The disk theorem for 4-manifolds, Proc. Internat. Congr. Math., 1984, North-Holland, New York, pp. 647-663.

[F2] - The topology of 4-manifolds, J. Differential Geom. 17 (1982), 357-453.

[H] J. F. P. Hudson, PL topology, Benjamin, New York, 1970.

[K] M. A. Kervaire, Le théorème de Barden-Mazur-Stallings, Comment. Math. Helv. 40 (1965), 31-42.

[KS] R. Kirby and L. C. Siebenmann, Foundational essays on topological manifolds, smoothing, and triangulations, Princeton Univ. Press, N.J., 1977.

[KwS] S. Kwasik and R. Schultz, Topological s-cobordisms of space-forms (to appear).

[MS] T. Matumoto and L. C. Siebenmann, The topological s-cobordism theorem in dimension four or five, Proc. Cambridge Philos. Soc. 84 (1968), 85-87.

[RS] C. P. Rourke and B. J. Sanderson, Introduction to piecewise linear topology, Exgeb. Math. Grenzgeb. Band 69, Springer-Verlag, New York, 1972.

[R] J. H. Rubinstein, On 3-manifolds which have finite fundamental group and contain Klein bottles, Trans. Amer. Math. Soc. 251 (1979) , 129-137.

[Sm] S. Smale, On the structure of manifolds, Amer. J. Math. 84 (1962), 387-399.

Department of Mathematics, Courant institute, New York UniverSITY, NEW YORK, NEW YORK 10012

Department of Mathematics, Rutgers University, New Brunswick, NEW JERSEY 08903 
